\title{
sciendo
}

\section{Making Decisions and Motor Actions with Technical Biomechanical Classifications in Male Judo Weight Categories}

\author{
by \\ Dany Alexis Sobarzo Soto ${ }^{1}$ Esteban Aedo-Muñoz ${ }^{2,3}$, Ciro José Brito ${ }^{4}$, Suzi Camey ${ }^{5}$, \\ Bianca Miarka ${ }^{4,6}$
}

The aim of the study was to compare motor action variables of judo combat phases and technical biomechanical assessment of the seven weight categories. The sample was composed of 638 bouts (176 of extra lightweight $<66 \mathrm{~kg}, 289$ of half lightweight $66>73 \mathrm{~kg}, 180$ of lightweight $73>81 \mathrm{~kg}, 244$ of half middleweight $81>90 \mathrm{~kg}, 174$ of middleweight $81>90 \mathrm{~kg}$, 151 of half heavyweight $90>100 \mathrm{~kg}$ and 142 of heavyweight $>100 \mathrm{~kg}$ ) during motor actions of approach, gripping, attack, defense and groundwork combat phases, verifying the interactions between them by Markov chains and comparisons by Kruskall-Wallis and Dunn post hoc tests $(p \leq .05)$. The results demonstrated that lighter weight categories showed higher approach phases than the heavyweight category, and the lightweight group demonstrated higher defensive action frequencies than the half lightweight category and heavyweight athletes. Heavyweight and middleweight categories demonstrated higher groundwork combat frequencies than lighter weights, and the heavyweight category showed higher pause frequencies than the lightweight category. Regarding the decision-making model, the main transitions presented by the Markov chains showed higher prevalence of the following combinations: gripping occurring before the lever and length attacks, lever attack followed by the length attack, and length attack occurring earlier than the groundwork phase.

Key words: martial arts, biomechanics, performance analysis, motor control, time motion analysis, circuit-based exercise.

\section{Introduction}

For all seven official categories (e.g., extra lightweight, half lightweight, lightweight, half middleweight, middleweight, half heavyweight and heavyweight), combat includes a complex interaction of multifaceted and open skills, requiring movements with refined control of many body parts performed in a constantly changing environment which may be unpredictable (Schimidt and Lee, 2013). Following time motion analysis, judo combats present irregular activity and pause patterns (Lech et al., 2015; Miarka et al., 2015; Santos et al., 2018; Maszczyk et al., 2018, 2020). Previous studies have revealed variables which influence motor actions in judo combats, including expertise (Drid et al.,

1 - Escuela de Kinesiologia. Universidad Santo Tomas, Santiago, Chile.

2 - Biomechanics Laboratory. Chilean High Performance Center. Physical Activity, Sport and Health Sciences Laboratory.

Universidad de Santiago de Chile, Chile.

3 - Physical Education Department. Universidad Metropolitana de Ciencias de la Educación, Santiago, Chile.

4 - Postgraduate Program in Physical Education. Physical Education Department. Federal University of Juiz de Fora, Brazil.

5 - Postgraduate Program in Epidemiology, School of Medicine. Statistic Department, Mathematic and Statistic Institute. Federal University of Rio Grande do Sul, Brazil.

6 - Postgraduate Program in Physical Education, School of Physical Education and Sports, Department of Fights. Federal University of Rio de Janeiro, Brazil. 
2015), the competitive outcome (Escobar-Molina et al., 2014; Maszczyk et al., 2018, 2020), the competitive level (Miarka et al., 2016, 2016a), weight categories (Brabec et al., 2019; Sterkowicz et al., 2013; Sterkowicz-Przybycien et al., 2017), the judging system and the effect of home advantage (Brito et al., 2017). Furthermore, age effects have been observed in the time dedicated to the approach, gripping, attack, groundwork and pause phases during judo competition (Miarka et al., 2014; Santos et al., 2018). Despite these performance analysis variances, the effects of motor actions during each combat phase have not been compared by the weight category. This knowledge would assist judo athletes in contextualizing training in their respective weight categories (Brabec et al., 2019; Coswig et al., 2018).

Studies have shown specific factors that affect judo performance and have suggested a determinant factor which could influence competitive success, such as the approach and grip attempts, gripping patterns (kumi kata), and effective attacks and their biomechanical levers (Calmet et al., 2010; Courel et al., 2014). Additionally, investigations about grip laterality and throwing side preferences have shown that attacks from the same gripping arrangement have better probabilities of resulting in an effective score for different weight categories using a diverse classification of attacks (Brito et al., 2017a; Kajmovic and Radjo, 2014).

Moreover, the taxonomy of typical judo throwing technique classification (Nage Waza) was created from a didactic requirement to group the techniques using an easier classification under a logical criteria of 1882 carried out by a proto biomechanical method. The standard techniques were categorized by body parts of Toris (the athlete who attacks), which work as larger contact points for energy transfer in throwing (i.e. Te waza $=$ shoulder, arm and hand techniques; Koshi waza $=$ hip techniques; Ashi waza $=$ leg techniques and Sutemi $w a z a=$ sacrifice techniques) (Sacripanti, 2012). An analysis according to biomechanical variables allows coaches to improve the contextualized techniques and to develop muscular strength and power of each execution (Gennadii et al., 2016; Miarka et al., 2017).

Gender comparisons of tactical interactions between penalties and subsequent attack effectiveness have shown fewer differences between male and female judo athletes (EscobarMolina et al., 2014). Thus, it is suggested that notational analysis in judo combats be directed with consideration toward weight category differences in each phase with a wide assortment of connected elements, such as the approach type (Calmet et al., 2010), the gripping type (Miarka et al., 2016), attack types (Sterkowicz et al., 2013), defensive actions (Boguszewski, 2011), and groundwork attempts (Brito et al., 2017a).

A recent study differentiated between four weight divisions by time motion analysis (Miarka et al., 2017). However, a varied motor action analysis examining each combat phase in all weight categories is required to evaluate the fighting skills and motor action pattern differences between official categories. This hypothesis is supported by the fact that physical (Sterkowicz-Przybycien and Almansba, 2011) and physiological (Sterkowicz-Przybycien and Fukuda, 2014; Torres-Luque et al., 2016) characteristics vary between and among these athletes. Therefore, the aim of the present study was to perform a notational analysis, a technical biomechanical assessment and statistical analyses of high level male judo combats of the seven weight categories on the following motor action variables: approach, gripping, defensive actions, attack and groundwork.

\section{Methods}

\section{Participants and design}

The present study considered high level judo athletes from each weight division who qualified for the Olympic Games in 638 competitive bouts divided according to weight divisions (176 extra lightweight $<66 \mathrm{~kg}$, 289 half lightweight $66>73 \mathrm{~kg}$, 180 lightweight $73>81 \mathrm{~kg}$, 244 half middleweight $81>90 \mathrm{~kg}, 174$ middleweight $81>90 \mathrm{~kg}, 151$ half heavyweight 90 $>100 \mathrm{~kg}$, and 142 heavyweight $>100 \mathrm{~kg}$ ). All bouts were analyzed to complete the motor actions and biomechanical aspects of techniques. The competitive bouts were analyzed using several publically available judo video databases in order to guarantee ecological validity and to verify the elite status of the sample, including those provided by the International Judo Federation (IJF) and the International Olympic Committee. Each video had to be of sufficient quality (standard definition 480/60i) and taken from a 
landscape view of the entire competition area in order to be included. The competitive bouts were evaluated following previously outlined protocols (Miarka et al., 2012; Miarka et al., 2018) from 36 international ranking competitions. The study was previously approved by the local Ethics and Research Committee.

\section{Procedures and measures}

Determination of movement with biomechanical and technical tactical patterns

The approach phase was subdivided into four categories according to the implemented movement pattern, including a right foot forward stance (Migi shizen hontai/Migi hontai), a left foot forward stance (Hidari shizen hontai/Hidari hontai), and a frontal stance (Shizen hontai/Jigo hontai) (Muddle et al., 2017). The domain attempt was evaluated by the attempt to grip with contact or by the location of the hand placement on the opponent's judo uniform (judogi) such as the collar, sleeve or back, and lateral location, right or left, following a previously validated protocol (Calmet et al., 2010).

The attack combat phase was characterized by specific biomechanical principles, which were identified by the type of the force couple applied or the length and application point of the movement arm, as outlined by Sterkowicz et al. (2013). Throwing techniques which employed a force couple were designated as using: an arm lever, an arm/foot lever, a trunk/leg lever, or a trunk/arm lever; while techniques described by the movement arm were designated as minimal length (applied at the opponent's waist), medium length (applied at the opponent's knee), variable length (below the opponent's waist) or maximal length (applied at the opponent's foot/ankle) (Sacripanti, 2012; Sterkowicz et al., 2013).

The defense in the standing phase was categorized by the manner in which the defending athlete changed his/her body position and orientation, right or left (Tae sabaki), in response to an attack and by the use of a counter attack (Miarka et al., 2017). The groundwork phase of combat was determined by the specific actions conducted, including defensive actions, immobilization/pinning techniques (Osae waza), chokes (Shime waza) and arm locks (Kansetsu waza) (Sacripanti, 2012; Sterkowicz et al., 2013).
Reliability testing

Reliability measures were carried out through intra and inter observer testing procedures to verify internal validity and guarantee the objectivity of the analysis. An agreement was classified as 'Almost Perfect' for Kappa values with a range of agreement between .84 and 1.0 for all combat phase frequency measures, with both being previously assessed using intra and inter observer tests (Miarka et al., 2011).

\section{Statistical Analysis}

Descriptive data are presented as median and mean $\left[25^{\text {th }}\right.$ percentile; $75^{\text {th }}$ percentile] values. The Markov process was used to describe how an athlete decided to move between a series of combat phase frequencies in continuous time, following preceding reports (Jackson, 2011; Miarka et al., 2018). Kruskal-Wallis followed by Dunn post hoc tests were conducted to compare motor actions and biomechanical analysis of technique frequencies between weight categories. A significance level of $p \leq .05$ was used. All analyses were conducted using SPSS 20.0 for Windows.

\section{Results}

Figure 1 shows a descriptive analysis of combat phases divided according to approach, gripping, attack, defense, groundwork and pause frequencies during a judo match.

Analysis presented significant differences of approach frequencies $\left(X^{2}=14.005, \mathrm{df}=6, p=\right.$ $.030)$, where lighter weight athletes had a higher frequency than the heavyweight athletes $(p=$ .021). Effects of gripping frequencies were observed $\left(X^{2}=19.470, \mathrm{df}=6, p=.003\right)$, in which the extra lightweight group had a lower number of gripping configurations than the lightweight ( $p=$ $.030)$, half middleweight $(p=.013)$, middleweight $(p=.002)$ and half heavyweight categories $(p=$ .036). No effect was observed in total attacks between weight categories $(p=.78)$. Comparisons indicated effects in defense frequencies $\left(X^{2}=2.704\right.$, $\mathrm{df}=6, p=.002)$, in which lightweight athletes used more defensive actions than those of the half lightweight $(p=.003)$ and heavyweight categories $(p=.009)$. Effects were observed in groundwork frequencies $\left(X^{2}=48.917, \mathrm{df}=6, p \leq .001\right)$, where the heavyweight category had higher frequencies than extra lightweight $(p \leq .001)$, half lightweight 
$(p \leq .001)$, lightweight $(p \leq .001)$ and half middleweight groups $(p \leq .001)$, while the middleweight category showed higher values than the lightweight $(p=.002)$ and half middleweight $(p=.032)$ categories. The comparisons of pause frequency showed effects $\left(X^{2}=18.313, \mathrm{df}=6, p=.005\right)$, in which the heavyweight category showed higher values than the lightweight category $(p \leq .001)$.

Figure 2 demonstrates the conditional probability of transition between combat states in different male judo weight categories.

Table 2 shows the frequencies of approach and gripping phases.

Comparisons of Migi shizen hontai/Migi hontai frequency showed differences $\left(X^{2}=57.917\right.$, $\mathrm{df}=6, p \leq .001)$, where the half lightweight category had lower values than extra lightweight $(p=.006)$ and half middleweight $(p \leq .001)$, while the half middleweight category had higher frequencies than the lightweight $(p \leq .001)$, middleweight $(p \leq .001)$, half heavyweight $(p \leq$ $.001)$ and heavyweight categories $(p=.006)$. Regarding Hidari shizen hontai/Hidari hontai frequency, analysis indicated differences $\left(X^{2}=\right.$ 5.944, $\mathrm{df}=6, p \leq .001)$, as the extra lightweight category had lower values than half lightweight $(p$ $=.002)$ and lightweight groups $(p=.036)$, while the half middleweight category presented lower frequencies than half lightweight $(p \leq .001)$ and lightweight ones $(p=.002)$, and the middleweight category had lower Hidari shizen hontai/Hidari hontai frequencies than half lightweight $(p \leq .001)$ and lightweight groups $(p=.014)$. Our analysis presented differences in Shizen hontai/Jigo hontai frequency $\left(X^{2}=76.449, \mathrm{df}=6, p \leq .001\right)$, as the half lightweight group had lower values than the half middleweight $(p \leq .001)$, middleweight $(p \leq .001)$, half heavyweight $(p=.042)$ and heavyweight category $(p \leq .001)$, and the lightweight category had lower values than half middleweight $(p=$ $.020)$, middleweight $(p \leq .001)$ and heavyweight categories $(p=.002)$, while the middleweight category had higher values of Shizen hontai/Jigo hontai than extra lightweight $(p \leq .001)$ and half middleweight groups $(p=.006)$.

The statistical analysis showed effects of trying to grip frequency $\left(X^{2}=3.412, \mathrm{df}=6, p \leq\right.$ $.001)$, in which half middleweight athletes tried to grip less than half lightweight $(p=.013)$ and lightweight $(p=.003)$, and half heavyweight athletes had lower attempts to grip than half lightweight $(p=.024)$ and lightweight $(p=.007)$. Also, heavyweight tried to grip less times than half lightweight $(p=.042)$ and lightweight groups $(p=.011)$. Comparisons showed significant differences of the right back grip when considering weight groups $\left(X^{2}=26.897, \mathrm{df}=6, p \leq\right.$ $.001)$, as half lightweight used the right back grip less times than half middleweight $(p \leq .001)$, middleweight $(p=.039)$ and heavyweight categories $(p=.002)$.

For right back and sleeve grip frequency, significant differences were found between weight categories $\left(X^{2}=13.120, \mathrm{df}=6, p=.041\right)$, where the half middleweight group demonstrated higher right back and sleeve grip frequencies than the half lightweight $(p=.026)$. Our analysis also demonstrated group differences in left back grip frequency $\left(X^{2}=21.242, \mathrm{df}=6, p=.002\right)$, as the extra lightweight group had lower left back frequencies than the lightweight group $(p=.022)$. Statistical analyses indicated differences in left back sleeve grip frequency between weight categories $\left(X^{2}=\right.$ 21.510, $\mathrm{df}=6, p \leq .001)$, however, no effects were observed in post hoc comparisons. Significant effects were observed $\left(X^{2}=17.170, \mathrm{df}=6, p=.009\right)$ for the right collar grip frequency, in which the extra lightweight category had lower right collar grip frequencies than the half heavyweight category $(p=.031)$.

Effects for right collar sleeve grip frequency were observed between weight categories $\left(X^{2}=18.553, \mathrm{df}=6, p=.005\right)$, as the heavyweight category used the right collar sleeve grip less times than the half heavyweight category $(p=.003)$. Comparisons showed differences of the left collar grip $\left(X^{2}=32.236, \mathrm{df}=6, p \leq .001\right)$, as the extra lightweight category used this grip less times than the lightweight $(p=.010)$ and half middleweight $(p=.0015)$, while the half lightweight had lower frequencies than the lightweight $(p \leq .001)$, half middleweight $(p=.003)$ and heavyweight groups $(p=.019)$. Regarding left collar and sleeve grip frequency, the comparisons showed significant differences $\left(X^{2}=48.103, \mathrm{df}=6\right.$, $p \leq .001)$, in which the half lightweight category demonstrated lower values than the lightweight $(p=.004)$, half middleweight $(p \leq .001)$ and heavyweight groups $(p=.009)$, and the half middleweight had higher values than middleweight $(p \leq .001)$ and half heavyweight 
categories $(p=.003)$.

For the collar grip frequency, comparisons showed differences between weight categories $\left(X^{2}=13.246, \mathrm{df}=6, p=.039\right)$ with no effects in the post hoc test. No effects were observed between groups on right sleeve grip comparisons $(p=.3)$. Comparisons showed significant differences of left sleeve grip frequency when comparing weight categories $\left(X^{2}=15.033, \mathrm{df}\right.$ $=6, p=.020)$, with no effects in the post hoc test. Our analysis showed effects on the left sleeve and right sleeve grip frequency $\left(X^{2}=39.862, \mathrm{df}=6, p \leq\right.$ $.001)$, as the extra lightweight category presented lower frequencies than the lightweight $(p=.002)$ and half middleweight group ( $p=.003)$, and the middleweight category presented higher frequencies than the lightweight $(p \leq .001)$ and half middleweight groups $(p \leq .001)$, while the heavyweight category had lower values than the half middleweight $(p=.048)$.

Table 3 shows the attempted and scored attacks, with biomechanical analysis of each technique used to perform the attack, defensive and groundwork phases.

Arm lever attempt comparisons revealed differences between weight categories $\left(X^{2}=16.555\right.$, $\mathrm{df}=6, p=.011$ ), as the heavyweight category demonstrated lower values than the lightweight group ( $p \leq .001)$. Also comparisons of arm and leg lever attempts presented differences $\left(X^{2}=22.478\right.$, $\mathrm{df}=6, p \leq .001)$, where the half middleweight category had higher values than the half lightweight $(p=.041)$, and the heavyweight category had higher frequencies than extra lightweight $(p=.041)$ and half middleweight categories $(p=.002)$. No effects were observed for the arm and leg lever scored $(p=.099)$.

Regarding the minimum lever, a significant difference was found between weight categories $\left(X^{2}=17.813, \mathrm{df}=6, p=.007\right)$, as the heavyweight category presented lower values than the half lightweight group $(p=.008)$. Significant differences were also observed when comparing the trunk and leg lever $\left(X^{2}=13.625\right.$, df $=6, p=.034)$, where the middleweight category had higher values than the half middleweight ( $p=$ .025), while comparisons for the trunk and leg lever scored had no effects between weight groups $(p=.120)$. Effects were observed when comparing waist lever attempts $\left(X^{2}=21.621, \mathrm{df}=\right.$ $6, p \leq .001)$, as the lightweight category demonstrated lower values than half heavyweight $(p=.023)$ and heavyweight categories $(p=.003)$. No effects were observed when comparing groups for the waist lever scored ( $p=.358)$.

Furthermore, effects were observed in maleolo attempts $\left(X^{2}=26.848, \mathrm{df}=6, p \leq .001\right)$, in which the heavyweight category demonstrated higher values than extra lightweight $(p=.018)$, half lightweight $(p=.014)$, lightweight $(p \leq .001)$ and half middleweight categories $(p \leq .001)$. Significant differences were also observed when comparing the maleolo lever scored $\left(X^{2}=16.214\right.$, $\mathrm{df}=6, p=.013)$, yet no effect was observed in post hoc tests. For defensive actions, significant differences between weight categories were observed $\left(X^{2}=2.704, \mathrm{df}=6, p=.002\right)$, as the lightweight category showed higher frequencies than half middleweight $(p=.003)$ and heavyweight categories $(p=.009)$. Regarding tae Sabaki to the left, comparisons showed significant differences between weight categories $\left(X^{2}=4.055\right.$, $\mathrm{df}=6, p \leq .001$ ), as the half middleweight category had lower values than half heavyweight $(p=.009)$ and middleweight groups $(p=.019)$, and higher than the lightweight category $(p \leq .001)$, while the heavyweight category showed higher values than lightweight $(p \leq .001)$ and middleweight categories $(p=.043)$. No effects were observed when comparing the counterattack $(p=.124)$ to Migi Tae Sabaki ( $p=.531)$. Significant differences were observed when comparing Kansetsu waza frequencies $\left(X^{2}=26.766, \mathrm{df}=6, p \leq .001\right)$, where the extra lightweight group had lower values than half lightweight $(p=.002)$, lightweight $(p=.002)$, middleweight $(p \leq .001)$ and heavyweight groups $(p \leq .001)$.

Significant differences were observed when comparing defensive actions $\left(X^{2}=49.854, \mathrm{df}=6, p\right.$ $\leq .001)$, in which the heavyweight category demonstrated higher values than extra light ( $p=$ .014), half lightweight ( $p \leq .001)$, lightweight ( $p \leq$ $.001)$, and half middleweight categories ( $p \leq .001)$, and the middleweight group had higher values than the lightweight $(p=.002)$ and half middleweight $(p=.036)$. Differences were noticed when comparing Shime waza $\left(X^{2}=14.290, \mathrm{df}=6, p\right.$ $=.027)$, as the half lightweight category showed lower values than the half heavyweight $(p=.030)$. No effects were observed when comparing weight categories for Osae waza ( $p=.226)$. However, significant effects were found $\left(X^{2}=24.855\right.$, df $=6$, 
$p \leq .001)$ when observing pauses, where the heavyweight category had higher values than lightweight $(p \leq .001)$ and half middleweight categories $(p=.003)$.

Table 1

Motor actions analysis of approach and gripping attempts of all weight categories.

\begin{tabular}{|c|c|c|c|c|c|c|c|}
\hline Groups & EL & HL & $\mathbf{L}$ & HM & $\mathbf{M}$ & HH & $\mathbf{H}$ \\
\hline \multicolumn{8}{|c|}{ Approach attempts } \\
\hline $\begin{array}{c}\text { Migi-shizen- } \\
\text { hontai/Migi-hontai }\end{array}$ & $2(.0 ; 7)^{*}$ & $.0(.0 ; 2)$ & $.0(.0 ; 5)^{@}$ & $4.5(.0 ; 11)^{*}$ & $1(.0 ; 4)^{@}$ & $.0(.0 ; 3)^{@}$ & $1(.0 ; 6)^{\circledR}$ \\
\hline $\begin{array}{c}\text { Hidari-shizen- } \\
\text { hontai/Hidari-hontai }\end{array}$ & $.0(.0 ; 4.5)$ & $4(.0 ; 1)^{@ \#}$ & $4(.0 ; 11.5))^{@ \#}$ & $.0(.0 ; 3)$ & $1(.0 ; 3)^{@ \# \& \&}$ & $1(.0 ; 8)$ & $.0(.0 ; 3)$ \\
\hline $\begin{array}{c}\text { Shizen-hontai/Jigo- } \\
\text { hontai }\end{array}$ & $.0(.0 ; 1.5)$ & $.0(.0 ; 1)$ & $.0(.0 ; 1)$ & $1(.0 ; 3) * \&$ & $3(.0 ; 11) * \& \# @$ & $1(.0 ; 6)^{*}$ & $1(.0 ; 4) * \&$ \\
\hline \multicolumn{8}{|c|}{ Gripping attemtps } \\
\hline Trying to grip & $8(3 ; 13)$ & $1(6 ; 15.8)$ & $11(6.8 ; 16)$ & $7.5(4 ; 12)^{* \&}$ & $9(4 ; 13)$ & $6(2.3 ; 12.8)^{* \& \varepsilon}$ & $7(4 ; 12)^{* \&}$ \\
\hline Right back & $14(8 ; 17.5)$ & $16.5(9 ; 26)$ & $19(12.8 ; 25.3)$ & $19(.0 ; 27.25)$ & $19(13 ; 29)^{*}$ & $21.5(8 ; 31)^{*}$ & $15(9 ; 26.5)^{*}$ \\
\hline Right back sleeve & $.0(.0 ; .0)$ & $.0(.0 ; .0)^{@}$ & $.0(.0 ; .0)$ & $.0(.0 ; .0)$ & $.0(.0 ; .0)$ & $.0(.0 ; .0)$ & $.0(.0 ; .0)$ \\
\hline Left back & $.0(.0 ; 1)$ & $.0(.0 ; 1)$ & $.0(.0 ; 1)^{\sharp}$ & $.0(.0 ; .0)$ & $.0(.0 ; 1)$ & $.0(.0 ; 0.8)$ & $.0(.0 ; 1)$ \\
\hline Left back sleeve & $.0(.0 ; .0)$ & $.0(.0 ; .0)$ & $.0(.0 ; .0)$ & $.0(.0 ; .0)$ & $.0(.0 ; .0)$ & $.0(.0 ; .0)$ & $.0(.0 ; .0)$ \\
\hline Right collar & $.0(.0 ; 1)$ & $.0(.0 ; 1)$ & $.0(.0 ; .3)$ & $.0(.0 ; 1)$ & $.0(.0 ; 2)$ & $.0(.0 ; 2)^{\#}$ & $.0(.0 ; 2)$ \\
\hline Right collar sleeve & $1(.0 ; 2)$ & $1(.0 ; 3)$ & $1(.0 ; 3)$ & $2(.0 ; 5)$ & $2(.0 ; 5)$ & $2(.0 ; 5)^{\%}$ & $1(.0 ; 3)$ \\
\hline Left collar & $.0(.0 ; 3)$ & $2(.0 ; 4)$ & $1(.0 ; 3)^{\sharp *}$ & $1(.0 ; 3)^{\sharp *}$ & $1(.0 ; 2)$ & $2(.0 ; 4.8)$ & $.0(.0 ; 2)^{*}$ \\
\hline Left collar sleeve & $.0(.0 ; 2)$ & $.0(.0 ; 2)$ & $1(.0 ; 5)^{*}$ & $2(.0 ; 4)^{*}$ & $1(.0 ; 4)^{@}$ & $1(.0 ; 3)^{\circledast}$ & $1(.0 ; 4)^{*}$ \\
\hline Collar collar & $1(.0 ; 5)$ & $.0(.0 ; 2)$ & $2(.0 ; 4)$ & $2(1 ; 5)$ & $1(.0 ; 2)$ & $1(.0 ; 2)$ & $1(.0 ; 5)$ \\
\hline Right sleeve & $.0(.0 ; 1)$ & $.0(.0 ; 1)$ & $.0(.0 ; 1)$ & $.0(.0 ; 1)$ & $.0(.0 ; 1)$ & $.0(.0 ; 2.8)$ & $.0(.0 ; 1)$ \\
\hline Left sleeve & $.0(.0 ; 1)$ & $.0(.0 ; 2)$ & $.0(.0 ; 1.3)$ & $1(.0 ; 2)$ & $.0(.0 ; 2)$ & $0.5(.0 ; 2)$ & $.0(.0 ; 2)$ \\
\hline Sleeve sleeve & $.0(.0 ; 1.5)$ & $1(.0 ; 3)$ & $1(.0 ; 2)^{\sharp}$ & $.0(.0 ; 1)^{\sharp}$ & $1(.0 ; 4)^{\# @ *}$ & $.0(.0 ; 2)$ & $.0(.0 ; 1.5)^{@}$ \\
\hline
\end{tabular}

Note $: \mu\left(1^{\circ} ; 3^{o}\right)-$ median $\left(25^{\circ} ; 75^{\circ}\right.$ percentiles $) E L=$ Extra Lightweight;

$H L=$ Half Lightweight $L=$ Lightweight $H M=$ Half Middleweight; $M=$ Middleweight;

$H H=$ Half Heavyweight; $H=$ Heavyweight. ${ }^{=}=$significant difference from the extra

lightweight category * = significant difference from the half lightweight category;

$@=$ significant difference from the half middleweight category; $\&=$ significant difference from

the lightweight category; \% significant difference from the heavyweight category,

$p \leq . .05$ for all comparisons. 
Table 2

Attempted and scored attacks classified from the biomechanical point of view, defensive and groundwork actions of all male weight categories.

Technical

analysis

EL

HL

L

HM

$\mathbf{M}$

HH

$\mathbf{H}$

Attempted and scored attacks

\begin{tabular}{|c|c|c|c|c|c|c|c|}
\hline Arm lever & $.0(.0 ; .0)$ & $.0(.0 ; .0)$ & $.0(.0 ; 1)$ & $.0(.0 ; .0)$ & $.0(.0 ; .0)$ & $.0(.0 ; .0)$ & $.0(.0 ; .0)$ \\
\hline Arm and leg lever & $2(.0 ; 3)$ & $2(.0 ; 4)$ & $1(.0 ; 4)$ & $2(1 ; 4)$ & $2(1 ; 6)$ & $2(1 ; 4)$ & $2(1: 4.5)$ \\
\hline $\begin{array}{c}\text { Arm and leg lever } \\
\text { with score }\end{array}$ & $.0(.0 ; .0)$ & $.0(.0 ; .0)$ & $.0(.0 ; .0)$ & $.0(.0 ; .0)$ & $.0(.0 ; .0)$ & $.0(.0 ; .0)$ & $.0(.0 ; .0)$ \\
\hline Minimum lever & $.0(.0 ; .0)$ & $.0(.0 ; 1)$ & $.0(.0 ; 1)$ & $.0(.0 ; 1)$ & $.0(.0 ; .0)$ & $.0(.0 ; 1)$ & $.0(.0 ; .0)$ \\
\hline Trunk leg lever & $.0(.0 ; 1)$ & $.0(.0 ; 2)$ & $.0(.0 ; 2)$ & $1(.0 ; 2)$ & $.0(.0 ; 1)$ & $.0(.0 ; 3)$ & $.0(.0 ; 2)$ \\
\hline $\begin{array}{l}\text { Trunk leg lever } \\
\text { with score }\end{array}$ & $.0(.0 ; .0)$ & $.0(.0 ; .0)$ & $.0(.0 ; .0)$ & $.0(.0 ; .0)$ & $.0(.0 ; .0)$ & $.0(.0 ; .0)$ & $.0(.0 ; .0)$ \\
\hline $\begin{array}{c}\text { Waist lever } \\
\text { variable }\end{array}$ & $1(.0 ; 2.5)$ & $.0(.0 ; 2)$ & $1(.0 ; 3)$ & $1(.0 ; 2)$ & $1(.0 ; 2)$ & $.0(.0 ; 1)$ & $.0(.0 ; 1)$ \\
\hline $\begin{array}{l}\text { Waist lever } \\
\text { variable with } \\
\text { score }\end{array}$ & $.0(.0 ; .0)$ & $.0(.0 ; .0)$ & $.0(.0 ; .0)$ & $.0(.0 ; .0)$ & $.0(.0 ; .0)$ & $.0(.0 ; .0)$ & $.0(.0 ; .0)$ \\
\hline Maleolo lever & $.0(.0 ; 2)$ & $.0(.0 ; 1)$ & $0.5(.0 ; 2)$ & $.0(.0 ; 2)$ & $1(.0 ; 2)$ & $.0(.0 ; 1)$ & $.0(.0 ; .0)$ \\
\hline $\begin{array}{c}\text { Maleolo lever } \\
\text { with score }\end{array}$ & $.0(.0 ; .0)$ & $.0(.0 ; .0)$ & $.0(.0 ; .0)$ & $.0(.0 ; .0)$ & $.0(.0 ; .0)$ & $.0(.0 ; .0)$ & $.0(.0 ; .0)$ \\
\hline
\end{tabular}

Defensive and groundwork actions

\begin{tabular}{|c|c|c|c|c|c|c|c|}
\hline Conterattack & $.0(.0 ; .0)$ & $.0(.0 ; 1)$ & $.0(.0 ; 1)$ & $.0(.0 ; .0)$ & $.0(.0 ; .0)$ & $.0(.0 ; 1)$ & $.0(.0 ; .0)$ \\
\hline $\begin{array}{c}\text { Tae Sabaki to the } \\
\text { Right }\end{array}$ & $1(.0 ; 3)$ & $1.5(.0 ; 3)$ & $2(.0 ; 4) \%$ & $1(.0 ; 3)$ & $1(.0 ; 5)^{* \& \%}$ & $1(.0 ; 3)^{*}$ & $2(.0 ; 3)$ \\
\hline $\begin{array}{c}\text { Tae Sabaki to the } \\
\text { Left }\end{array}$ & $2(.0 ; 4)$ & $1(.0 ; 3)$ & $2(1 ; 4)$ & $1(.0 ; 2)$ & $2(.0 ; 3)$ & $2(0.3 ; 4)$ & $1(.0 ; 2)$ \\
\hline Osae-waza & $.0(.0 ; .0)$ & $.0(.0 ; .0)$ & $.0(.0 ; .0)$ & $.0(.0 ; .0)$ & $.0(.0 ; .0)$ & $.0(.0 ; .0)$ & $.0(.0 ; .0)$ \\
\hline Kansetsu-waza & $.0(.0 ; .0)$ & $.0(.0 ; .0)^{\#}$ & $.0(.0 ; .0)^{\#}$ & $.0(.0 ; .0)$ & $.0(.0 ; .0)^{\#}$ & $.0(.0 ; .0)$ & $.0(.0 ; .0)^{\#}$ \\
\hline Shime-waza & $.0(.0 ; .0)$ & $.0(.0 ; .0)$ & $.0(.0 ; .0)$ & $.0(.0 ; .0)$ & $.0(.0 ; .0)$ & $.0(.0 ; .0)^{*}$ & $.0(.0 ; .0)$ \\
\hline Passive position & $5(2 ; 9) \%$ & $5(3 ; 9.8)^{\%}$ & $7.0(4 ; 11) \%$ & $\begin{array}{c}6.0(2 ; 11) \\
\%\end{array}$ & $\begin{array}{c}4(2 ; 7.0) \\
\text { \%@\& }\end{array}$ & $5(1 ; 8)$ & $3(1 ; 5)$ \\
\hline
\end{tabular}

Note: $\mu\left(1^{\circ} ; 3^{o}\right)-$ median $\left(25^{\circ} ; 75^{\underline{o}}\right.$ percentiles $) E L=$ Extra Lightweight;

$H L=$ Half Lightweight $L=$ Lightweight; $H M=$ Half Middleweight; $M=$ Middleweight; $H H=$ Half Heavyweight; $H=$ Heavyweight. ${ }^{*}=$ significant difference from the extra lightweight category; ${ }^{*}=$ significant difference from the half lightweight category; $\mathbb{Q}=$ significant difference from the half middleweight category; ${ }^{*}=$ significant difference from the lightweight category; \% = significant difference from the heavyweight category, $p \leq .05$ for all comparisons. 


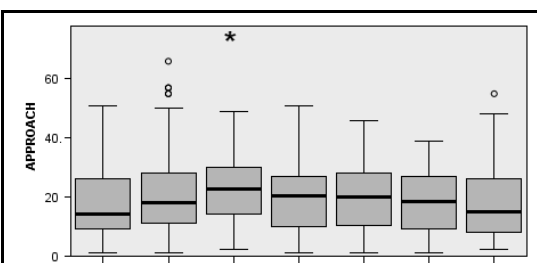

EL HL L HM M HH H

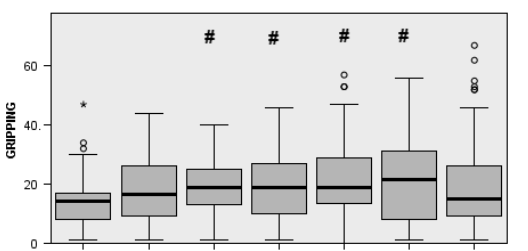

EL HL L HM M HH H

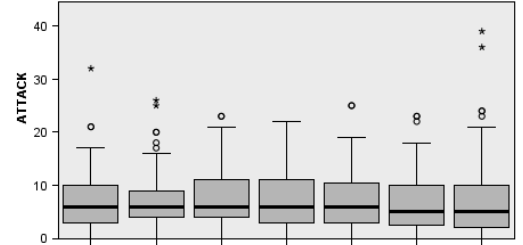

EL HL L HM M HH H

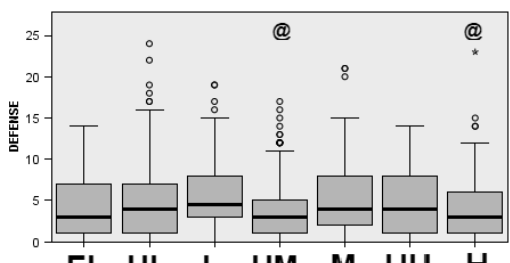

EL HL L HM M HH $\mathrm{H}$
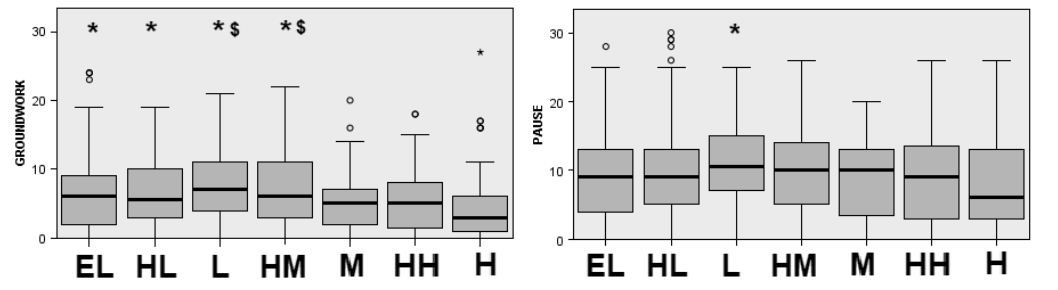

Figure 1

Box-plot of combat phases (frequencies).

Note: $\mathrm{EL}$ = extra-lightweight; $\mathrm{HL}$ = half-lightweight; $\mathrm{L}=$ lightweight;

$\mathrm{HM}=$ half-middleweight; $\mathrm{M}=$ middleweight; $H H=$ half-heavyweight;

$H=$ heavyweight; * = significant difference of the heavyweight category;

\# = significant difference of the lightweight category; @ = significant difference of the

lightweight category; \$ = significant difference of the middleweight category,

$$
p \leq .05 \text { for all comparisons. }
$$

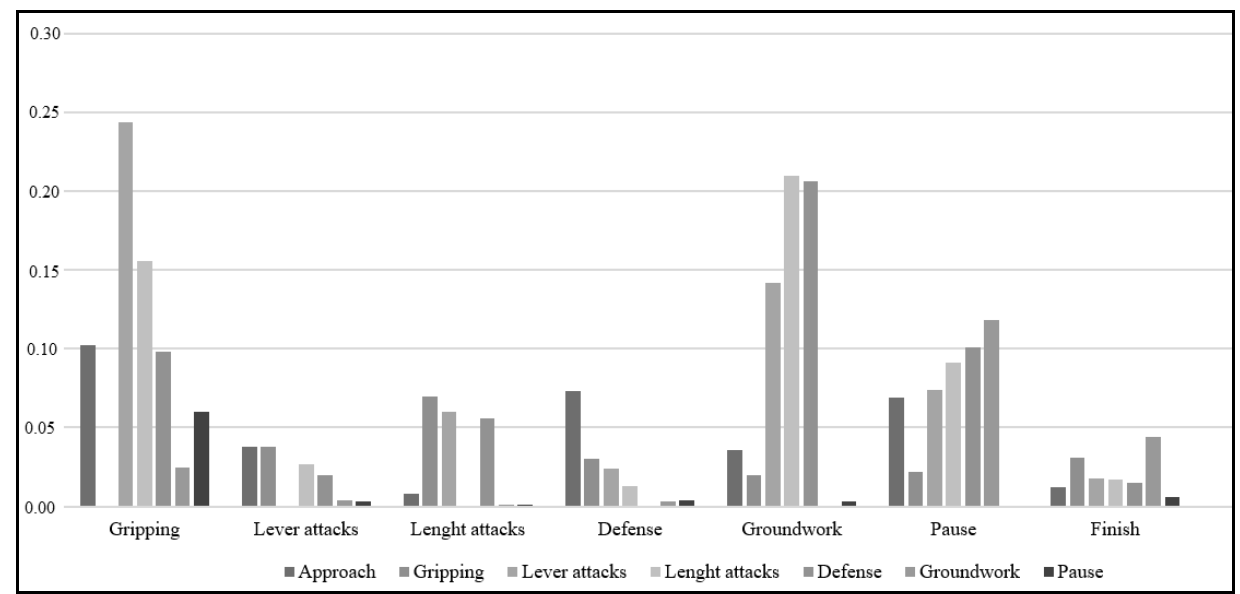

Figure 2

Conditional probability of transition between combat phases.

Note: Order of the correlations between phases is related to that described in the legend - the first column is related to the correlation with the approach phase, the second with gripping, the third with the lever attacks, then length attacks, defense, groundwork and pause. The correlation column with the phase itself was removed. No effects between conditional probabilities of transition between combat phases were observed when compared weight categories, $p \leq .05$ for all comparisons. 


\section{Discussion}

The present study analyzed all the seven weight categories of male athletes considering the motor action variables of approach, gripping, defensive actions, attack (their biomechanical analysis) and groundwork. The results demonstrated that lighter weight categories showed higher approach phases than the heavyweight category, and the lightweight group demonstrated higher defensive action frequencies than the half lightweight category and heavyweight athletes. Heavyweight and middleweight categories demonstrated higher groundwork combat frequencies than lighter weights, and the heavyweight category showed higher pause frequencies than the lightweight category. Regarding the decision-making model, the main transitions presented by the Markov chains showed higher prevalence of the following combinations: gripping occurring before the lever and length attacks, lever attack followed by the length attack, and length attack occurring earlier than the groundwork phase.

Regarding the approach phase, high frequencies could be used by athletes to engage in evasive actions in order to control space and ultimately gain control of their opponent for subsequent attacks (Brito et al., 2017a; Coswig et al., 2018; Miarka et al., 2017). The present results show that the extra lightweight category had a lower frequency of the left antero-posterior dominant position (Hidari shizen hontai/Hidari hontai) than the other weight categories, while the half middleweight category presented lower values of the right antero-posterior and frontal dominant positions (Migi shizen hontai/Migi hontai and Shizen hontai/Jigo hontai, respectively). Athletes of both categories rapidly analyze their adversary and execute their anticipated gripping tactics, which has been recognized to be a key factor in technical efficacy (Imamura et al., 2007; Santos et al., 2017). However, a possible explanation for these differences presented in the results is the fact that the half middleweight group has a greater number of athletes in absolute volume participating in judo tournaments than the extra lightweight. Therefore, half middleweight athletes have to develop unpredictable or difficult forms of approach, avoiding Migi shizen hontai/Migi hontai and Shizen hontai/Jigo hontai positions which are more typical and vulnerable positions.

Regarding the gripping phase, there is a range of primary and secondary factors influencing the development of judo expertise, and gripping appears to be one of the essential characteristics that determines proficiency in competition (Durana et al., 2018; Sterkowicz et al., 2010). The present study showed that middleweight athletes had higher gripping frequencies than other weight categories, particularly gripping more often with one hand targeting their opponent's back and sleeves. Recent findings by Muddle et al. (2017) outline the relationship between balance control and the bilateral reactionary gripping task. It is possible that middleweight athletes use a varied number of grip combinations to avoid opponent attacks and to execute throwing techniques with the purpose of compromising their opponent's balance and causing them to fall to the ground (Imamura et al., 2006; Miarka et al., 2016; Muddle et al., 2017). In addition, heavier athletes used more defensive grip configurations (i.e. back and collars) aiming to control their dynamic posture, and at the same time avoiding opponent's possible attempts to displace their balance (center of gravity), approaches and arm lever or minimal length techniques (Imamura et al., 2006; Muddle et al., 2017).

With respect to attacks and defensive phases, the present study agrees with preceding ones that showed lower extremity techniques were generally more positively combined by middle and heavier athletes, while maximal length (i.e. sacrifice) techniques tended to be executed more frequently by smaller and lighter competitors who presented greater agility (Imamura et al., 2006, 2007; Sacripantti, 2012). The heavyweight category showed higher frequencies of anticipatory defensive actions (Tae Sabaki) to the left than lightweight and middleweight categories, which could be associated with a high number of attacks to the right connected with the dominance by the right collar grip, as shown by our data.

The present results showed that middleweight athletes had more effective techniques with arm and leg levers than the lighterweight and heavier judo athletes. Lower extremity techniques (ashi waza) executed to the front or side (left/right), such as o soto gari and de 
ashi haraí, require high amounts of relative torque/weight before realizing the opponent's imbalance (kuzushi), compared to those executed to the rear orientation which involve rotation such as morote seoi nage, and sacrifice techniques (sutemi waza) such as tomoe nage (Imamura et al., 2006, 2007). The use of sacrifice attacks has been reported to happen more frequently in senior than junior athletes (Boguszewski, 2011), and may be chosen during high level judo tournaments due to better scoring (Sacripantti, 2012).

Groundwork actions appear to be related to attacking actions during standing combat and $20 \%$ of all attack attempts during the 2012 Olympic Games occurred during the transition to/or into groundwork (Miarka et al., 2016a). In the present study, heavier and middleweight athletes presented more frequent attacks and a greater use of armlocks and choking techniques than lightweight athletes. The heavyweight category also demonstrated a higher defensive position than other weight categories, and these data may suggest that attacks by heavier athletes did not result in match ending (ippon) scores. In support of this, fewer ippon are scored and a higher number of pauses are associated with heavier athletes during international competition, which suggests that heavier elite athletes tactically place their adversaries in susceptible positions through the transition to groundwork (Miarka et al., 2016a). All this contextual information about weight category variances can be used to support motor action improvement efforts, which are the main goal of any judo development program for high level athletes.

\section{Conclusion}

The present research examined all seven weight categories of judo male combats on the frequency and interaction between combat phases (i.e. approach, gripping, defensive actions, attack and their biomechanical analyses, and groundwork) and their motor actions. Markov chains showed higher prevalence of combinations for all weight categories which included gripping occurring before the lever and length attacks, lever attack followed by the length attack, and length attack occurring before the groundwork phase. Regarding combat phase frequencies, heavier and lighter athletes demonstrated singularities and solid definitions of approach, gripping, attack and defensive actions, while middleweight athletes showed more grip configurations and variable unpredictable attacks. These outcomes may be applied in intermittent training with specific biomechanical demands in conjunction with current essential information of combat sport psychology and physiology as a means for assisting in judo training, preparation and competition.

\section{References}

Boguszewski D. Defensive actions of world top judoists. J Hum Kinet, 2011; 27: 111-122

Brabec L, Bello FD, Araujo RA, Brito CJ, Fernandes JR, Miarka B. Judo approach and handgrip analysis: determining aspects of world circuit high performance. J Physic Educ Sport, 2019; 19: 413-419

Brito CJ, Miarka B, de Durana, ALD, Fukuda, DH. Home Advantage in Judo: Analysis by the Combat Phase, Penalties and the Type of Attack. J Hum Kinet, 2017; 57(1): 213-220

Brito CJ, Moreira DG, Fernandes JR, Ferreira AP, Cordova C, Miarka B. Technical-tactical comparisons between weight divisions in elite judo athletes. Rev Port Cie Desp, 2017a; 49: 49-67

Calmet M, Miarka B, Franchini E. Modeling of grasps in judo contests. Int J Perform Anal Sport, 2010; 10: 229240

Coswig VS, Gentil P, Bueno JCA, Follmer B, Marques VA, Del Vecchio FB. Physical fitness predicts technicaltactical and time-motion profile in simulated Judo and Brazilian Jiu-Jitsu matches. PeerJ, 2018; 25: 6:e4851

Courel J, Franchini E, Femia P, Stankovic N, Escobar Molina R. Effects of kumi kata grip laterality and throwing side on attack effectiveness and combat result in elite judo athletes. Int J Perform Anal Sport, 2014; 14: 138-147 
Drid P, Casals C, Mekic A, Radjo I, Stojanovic M, Ostojic SM. Fitness and anthropometric profiles of international vs. national judo medalists in half heavyweight category. J Strength Cond Res, 2015; 29: 2115-2121

Durana ALD, Bello FD, Brito CJ, Miarka B. High level performance in world judo circuit: notational analyzes of combat phase by weight categories. J Hum Sport Exe, 2018; e-print

Escobar-Molina R, Courel J, Franchini E, Femia P, Stankovic N. The impact of penalties on subsequent attack effectiveness and combat outcome among high elite judo competitors. Int J Perform Anal Sport, 2014; 14: $946-954$

Gennadii A, Sergii I, Pavol B, Mykola N, Wojciech CJ. The use of didactic laws in the teaching of the physical elements involved in judo techniques. Ido Mov Cult, 2016; 16(4): 21-30

Imamura RT, Hreljac A, Escamilla RF, Edwards B. A three dimensional analysis of the center of mass three different judo throwing techniques. J Sports Sci Med, 2006; CSSI: 122-131

Imamura RT, Iteya M, Hreljac A, Escamilla RF. A kinematic comparison of the judo throw Harai Goshi during competitive and non competitive conditions. J Sports Sci Med, 2007; CSSI: 12-22

Jackson CH. Multi state models for panel data: the msm package for R. J Stat Software, 2011; 38(8): 1-29

Kajmovic H, Radjo I. A comparison of Gripping Configuration and Throwing Techniques Efficiency Index in Judo between Male and Female Judoka during Bosnia and Herzegovina Senior Phase Championships. Int J Perform Anal Sport, 2014; 14: 620-634

Lech G, Chwala W, Ambrozy T, Sterkowicz S. Muscle torque and its relation to technique, tactics, sports level and age group in judo contestants. J Hum Kinet, 2015; 7: 167-175

Maszczyk A, Gołaś A, Pietraszewski P, Kowalczyk M, Cięszczyk P, Kochanowicz A, Smółka W, Zając A. Neurofeedback for the enhancement of dynamic balance of judokas. Biol. Sport 2018; 35: $99-102$

Maszczyk A, Dobrakowski P, Nitychoruk M, Żak M, Kowalczyk M, Toborek M. The Effect of Neurofeedback Trainingon the Visual Processing Efficiency in Judo Athletes. J Hum Kinet, 2020; 71: 219-227

Miarka B, Branco BH, Del Vecchio FB, Calmet M, Franchini E. Development and validation of a time motion judo combat model based on the Markovian Processes. Int J Perform Anal Sport, 2015; 15: 315-331

Miarka B, Brito CJ, Amtmann J, Cordova C, Bello FD, Camey S. Suggestions for judo training with pacing strategy and decision making by judo championship phases. J Hum Kinet, 2018, 61: 219-232

Miarka B, Cury R, Julianetti R, Battazza R, Julio UF, Calmet M, Franchini E. A comparison of time-motion and technical-tactical variables between age groups of female judo matches. J Sports Sci, 2014, 32(16): 1529-38

Miarka B, Fukuda DH, Del Vecchio FB, Franchini E. Discriminant analysis of technical tactical actions in high level judo athletes. Int J Perform Anal Sport, 2016; 16(1): 30-39

Miarka B, Fukuda HD, Heinisch HD, Battazza R, Del Vecchio FB, Camey S, Franchini, E. Time-motion analysis and Decision Making in Female Judo Athletes during Victory or Defeat at Olympic and Non Olympic Events: Are Combat Actions Really Unpredictable? Int J Perform Anal Sport, 2016a; 16(2): 442463

Miarka B, Hayashida CR, Julio UF, Calmet M, Franchini E. Objectivity of FRAMI software for judo match analysis. Int J Perform Anal Sport, 2011; 11: 254-266

Miarka B, Panissa VLG, Julio UF, Del Vecchio FB, Calmet M, Franchini E. A comparison of time motion performance between age groups in judo matches. J Sports Sci, 2012; 30(9): 899-905

Miarka B, Sterkowicz-Przybycien, K, Fukuda DH. Evaluation of Sex Specific Movement Patterns in Judo Using Probabilistic Neural Networks. Mot Control, 2017; 21(4): 390-412

Muddle TWD, Fukuda DH, Wang R, Riffe JJ, Church DD, Beyer KS, Hoffman JR, Stout JR. Effects of a 10 Week Introductory Judo Course on Postural Control During a Bilateral Reactionary Gripping Task. Mot Control, 2017; 21(4): 373-389 
Sacripanti A. Biomechanical revision of the principles of Dr. Jigoro Kano's judo kodokan. Med Del Sport, 2012; 65(2): 265-281

Santos L, Fernández-Río J, Iglesias-Soler E, Blanco-Traba M, Jakobsen MD, González-Díez V, Franchini E, Gutiérrez C, Dopico-Calvo X, Carballeira-Fernández E, Amonette W, Suman O. Postural control and physiological responses to a simulated match in U-20 judo competitors. Sports Biom, 2017; 21: 1-14

Schimidt RA, Lee TD. Motor Learning and Performance. Champaign: Human Kinetics, 336; 2013

Sterkowicz S, Lech G, Blecharz J. Effects of laterality on the technical/tactical behavior in view of the results of judo fights. Arch Budo, 2010; 6: 173-177

Sterkowicz S, Sacripanti A, Sterkowicz-Przybycien KL. Techniques frequently used during London Olympic judo tournaments: a biomechanical approach. Arch Budo, 2013; 1: 51-58

Sterkowicz-Przybycien KL, Almansba R. Sexual dimorphism of anthropometrical measurements in judoists vs untrained subject. Sci Sports, 2011; 26: 316-323

Sterkowicz-Przybycien KL, Fukuda DH. Establishing normative data for the special judo fitness test in female athletes using systematic review and meta analysis. J Strength Cond Res, 2014; 28: 3585-3593

Torre-Luque G, Hernández-García R, Escobar-Molina R, Garatachea N, Nikolaidis PT. Physical and Physiological Characteristics of Judo Athletes: An Update. Sports, 2016; 4(1): 20

\section{Corresponding author:}

\section{Bianca Miarka}

Faculdade de Educacao Fisica e Desporto - FAEFID

José Lourenço Kelmer st. - Martelos, Juiz de Fora - MG, Brazil. ZIP. 36036-330

Telephone: +55(32) 2102-3911,

FAX +55(32) 2102-3989.

E-mail: miarkasport@hotmail.com 